\title{
Feasibility of Charcoal Tattooing of Cytology-Proven Metastatic Axillary Lymph Node at Diagnosis and Sentinel Lymph Node Biopsy after Neoadjuvant Chemotherapy in Breast Cancer Patients
}

\author{
Seho Park, MD, PhD',2 \\ Ja Seung Koo, MD, PhD 3 \\ Gun Min Kim, MD ${ }^{4}$ \\ Joohyuk Sohn, MD, PhD ${ }^{4}$ \\ Seung II Kim, MD, PhD' \\ Young Up Cho, MD, PhD' \\ Byeong-Woo Park, MD, PhD' \\ Vivian Youngjean Park, MD, PhD ${ }^{5}$ \\ Jung Hyun Yoon, MD, PhD 5 \\ Hee Jung Moon, MD, PhD 5 \\ Min Jung Kim, MD, $P D^{5}$ \\ Eun-Kyung Kim, MD, PhD 5
}

\begin{abstract}
${ }^{1}$ Division of Breast Surgery, Department of Surgery, Yonsei University College of Medicine, Seoul, ${ }^{2}$ Frontier Research Institute of Convergence Sports Science,

Yonsei University, Seoul, ${ }^{3}$ Department of

Pathology, Yonsei University College of

Medicine, Seoul, ${ }^{4}$ Division of Medical

Oncology, Department of Internal Medicine, Severance Hospital, Yonsei University College of Medicine, Seoul, ${ }^{5}$ Department of Radiology and Research Institute of Radiological Science, Severance Hospital, Yonsei University College of Medicine, Seoul, Korea
\end{abstract}

Correspondence: Min Jung Kim, MD, PhD Department of Radiology and Research Institute of Radiological Science, Severance Hospital, Yonsei University College of Medicine,

50-1 Yonsei-ro, Seodaemun-gu, Seoul 03722, Korea Tel: $82-2-2228-7400$

Fax: 82-2-393-3035

E-mail: mines@yuhs.ac

Received May 2, 2017

Accepted August 7, 2017

Published Online August 17, 2017

*Seho Park and Ja Seung Koo contributed equally to this work.

\begin{abstract}
Purpose
Sentinel lymph node biopsy (SLNB) can be performed when node-positive disease is converted to node-negative status after neoadjuvant chemotherapy (NCT). Tattooing nodes might improve accuracy but supportive data are limited. This study aimed to investigate the feasibility of charcoal tattooing metastatic axillary lymph node (ALN) at presentation followed by SLNB after NCT in breast cancers.
\end{abstract}

\section{Materials and Methods}

Twenty patients with cytology-proven node metastases prospectively underwent charcoal tattooing at diagnosis. SLNB using dual tracers and axillary surgery after NCT were then performed. The detection rate of tattooed node and diagnostic performance of SLNB were analyzed.

\section{Results}

All patients underwent charcoal tattooing without significant morbidity. Sentinel and tattooed nodes could be detected during surgery after NCT. Nodal pathologic complete response was achieved in 10 patients. Overall sensitivity, false-negative rate (FNR), negative predictive value, and accuracy of hot/blue SLNB were $80.0 \%, 20.0 \%, 83.3 \%$, and $90.0 \%$, respectively. Retrieving more nodes and favorable nodal response were associated with improved performance. The best accuracy was observed when excised tattooed node was calculated together (FNR, 0.0\%). Cold/non-blue tattooed nodes of five patients were removed during non-sentinel axillary surgery but clinicopathological parameters did not differ compared to patients with hot/blue tattooed node detected during SLNB, suggesting the importance of the tattooing procedure itself to improve performance.

\section{Conclusion}

Charcoal tattooing of cytology-confirmed metastatic ALN at presentation is technically feasible and does not limit SLNB after NCT. The tattooing procedure without additional preoperative localization is advantageous for improving the diagnostic performance of SLNB in this setting. 


\section{Introduction}

Sentinel lymph node biopsy (SLNB) is a standard procedure for managing patients with clinical node-negative breast cancer and it lowers surgical morbidity and improves quality of life compared to axillary lymph node dissection (ALND) while providing similar survival rates $[1,2]$. However, the oncologic safety of SLNB remains controversial in patients with cytology-proven node-positive disease at presentation that is converted to clinical node-negative status after receiving neoadjuvant chemotherapy (NCT). Nodal metastasis can be eradicated by NCT in up to approximately $40 \%$ of patients with positive axillary lymph node (ALN) metastasis at diagnosis [3,4], but the possible effects of NCT are alterations or disruptions of lymphatic vessels or nonuniform tumor regression of metastatic ALNs, which can limit the usefulness of SLNB in these patients [5].

Several prospective clinical trials were undertaken to determine the accuracy of SLNB after NCT in patients with clinical or histological node-positive disease and the overall false-negative rate (FNR) of SLNB was $14.2 \%$ in the SENTinel NeoAdjuvant (SENTINA) study, $12.6 \%$ in the American College of Surgeons Oncology Group (ACOSOG) Z1071 (Alliance) trial, and $13.3 \%$ in the Sentinel Node Biopsy Following Neoadjuvant Chemotherapy (SN FNAC) trial [6-8]. In cases where dual tracers were used, $>2$ sentinel lymph nodes (SLNs) were retrieved, or immunohistochemistry was used; however, FNRs $<10 \%$ were clinically acceptable for SLNB [4]. Thus, the National Comprehensive Cancer Network (NCCN) guidelines recommend SLNB or ALND if biopsyproven metastatic ALNs are converted to clinically nodenegative status after preoperative systemic therapy [9]. Importantly, the NCCN guidelines emphasize that marking sampled ALNs with a clip or tattoo before NCT can improve the diagnostic performance of SLNB [10,11].

A biopsy-proven metastatic ALN has to be marked if targeted axillary dissection is to be performed after NCT and this marking has to take place by an experienced radiologist with specialized equipment such as a clip [12]. Preoperative localization with a wire or radioactive seed is also necessary and this additional invasive procedure might be uncomfortable for patients. Furthermore, clinicians must also check possible clip migration and determine the radiation safety of the radioactive seed [4]. In comparison, additional localization is not required for the tattooing technique of positive node although limited studies have been reported since its introduction [11]. The diagnostic performance of the tattooing procedure incorporated into SLNB should be studied with this background in mind. In addition, marked metastatic ALN before NCT is not always detected as hot and/or blue nodes at surgery. For clarifying the association between tattooed ALN at diagnosis and dual traced nodes after NCT, SLNB was considered as results of hot/blue ALNs retrieved alone and modified SLNB was defined as findings of dual traced or residual marked, suspiciously enlarged nodes in this study.

The main aims of the present study were primarily to investigate whether black tattoo injected in cytology-proven metastatic ALN at presentation could be detected intraoperatively in patients treated with NCT and to examine the feasibility of charcoal tattooing in breast cancers. The second was to evaluate the diagnostic performance of SLNB with or without marking the biopsy-proven metastatic ALN by calculating the detection rate, sensitivity, FNR, negative predictive value (NPV), and accuracy. The last was to determine the concordance between charcoal-tattooed ALN and SLNs detected by radioisotopes and blue dye. If a discordance was found, we tried to identify which clinicopathological parameters were associated with the discordance.

\section{Materials and Methods}

\section{Patient enrollment}

Twenty patients with clinical T1-T3 breast cancer at diagnosis who received NCT between October 2015 and July 2016 were prospectively enrolled from Yonsei Cancer Center, Severance Hospital, Yonsei University College of Medicine, Seoul, Korea. All patients had cytology-proven ALN metastases prior to NCT. Patients with a past history of allergic reaction to charcoal agents, inflammatory or T4 breast carcinoma, or stage IV cancer at diagnosis were excluded. At presentation, all patients with clinically enlarged ALNs or with radiologically suspicious nodal findings of loss of fatty hilum, cortical thickening $>3 \mathrm{~mm}$, round shape, markedly hypoechoic cortex, or increased peripheral blood flow underwent ultrasound-guided fine needle aspiration cytology of the most suspicious or largest ALN to confirm the presence of metastasis.

\section{Ultrasound-guided charcoal tattooing on cytology-con- firmed ALN}

Prior to NCT, the main breast tumor was marked with a metallic clip and then the cytology-proven metastatic ALN was tattooed with charcoal suspension (Charcotrace, Phebra, Sydney, Australia) through ultrasound-guidance by a radiologist (M.J.K.) who had more than 14 years of specialized experience in breast imaging. To minimize obstacles to pathologic examination, the charcoal was mainly injected 
into the capsular area of the metastasis-confirmed ALN and a needle-point injection track was made from the ALN to the skin of the axilla. A median dose of $0.5 \mathrm{~mL}$ (range, 0.2 to 0.8 $\mathrm{mL}$ ) was used for node tattooing.

\section{Response to NCT and clinicopathological factors}

After four cycles of anthracycline followed by taxane-containing NCT, the responses of the primary tumor and nodes were comprehensively evaluated using clinical and radiological examinations, mainly with ultrasound or breast magnetic resonance images. For cases with human epidermal growth factor receptor 2 (HER2)-positive disease, trastuzumab was incorporated into the NCT regimens. According to the Response Evaluation Criteria in Solid Tumors ver. 1.1, tumor response to NCT was considered as complete or partial response and non-response was defined with stable or progressive disease. The ALN response to NCT was defined with the disappearance of metastatic ALNs or no suspicious imaging findings in the axilla. Nodal non-response was considered when suspicious findings in the axilla remained after completion of NCT.

Pathologic stages were based on the AJCC Cancer Staging Manual, seventh edition [13]. The modified Scarff-BloomRichardson grading system was used for histologic grading. Tumors with $\geq 1 \%$ nuclear-stained cells by immunohistochemistry using core needle biopsy specimens were considered positive for estrogen receptor (ER) and progesterone receptor (PR) according to the American Society of Clinical Oncology/College of American Pathologists (ASCO/CAP) guidelines [14]. HER2 staining was scored as $0,1+, 2+$, or $3+$ according to the ASCO/CAP guidelines [15]. In cases with HER2 2+ results, silver in situ hybridization (SISH) was performed using the INFORM HER2 Dual ISH DNA Probe Cocktail Assay (Ventana Medical Systems, Tucson, AZ) with an automated slide stainer according to the manufacturer's protocols. HER2 gene amplification was defined with a HER2 gene/ chromosome 17 copy number ratio $\geq 2.0$ or a HER2 gene/ chromosome 17 copy number ratio $<2.0$ with an average HER2 copy number $\geq 6.0$ signals/ cell according to the ASCO/CAP guidelines [15]. An immunohistochemistry score of $3+$ or gene amplification by SISH was considered HER2-positive. During the study period, Ki-67 staining was mainly performed on post-treatment surgical specimens for residual carcinomas in our institution and was not investigated in the present study.

Based on ER, PR, HER2, and histologic grade findings, molecular phenotypes were categorized into the following four subgroups: luminal A (ER-positive and/or PR-positive, HER2-negative and grade 1 or 2), luminal B (ER-positive and / or PR-positive, HER2-negative and grade 3; or ER-positive and/or PR-positive, and HER2-positive), HER2- enriched (ER-negative, PR-negative, and HER2-positive), and triple-negative breast cancer (TNBC) (ER-negative, PR-negative, and HER2-negative).

\section{SLNB, axillary surgery, and pathologic assessment}

SLNs were detected using a combination of radioisotopes and blue dye techniques except for one detected with a radioisotope alone. In brief, $0.5 \mathrm{mCi}{ }^{99 \mathrm{~m} T c-P h y t a t e ~(K o r e a ~}$ Atomic Energy Research Institute, Daejeon, Korea) was diluted in $0.5 \mathrm{~mL}$ saline and injected into the subcutaneous layer of the areolar tissue in the direction of the main primary tumor at the Department of Nuclear Medicine on the day of surgery. In the operation room just before surgery was initiated, $0.8 \%$ indigo carmine dye (Carmine, Korea United Pharm Inc., Seoul, Korea) was injected into the periareolar area followed by a massage of the injection site. SLNs were excised and defined as hot and/or blue-colored nodes with a handheld gamma probe (Neoprobe Gamma Detection System, Neoprobe Corp., Dublin, $\mathrm{OH}$ ) at gross visual inspection or with a radioactive count of $\geq 10 \%$ of the ex vivo count of the hottest node. During SLNB, clinically palpable cold or non-blue axillary nodal tissues were also excised and categorized as axillary sampling. Charcoal-tattooed ALN and track were removed during axilla surgery and included in the SLNB or axillary sampling procedures. SLNs and axillary sampling tissues were then sent to frozen section analysis. After SLNB and / or axillary sampling, the decision to perform further concomitant completion level I/II ALND was made taking into account patient' consent, initial nodal burden, node responses during and after NCT, or frozen section results of SLNB and / or axillary sampling.

All excised ALNs including SLNs were subjected to routine pathological examinations, which included hematoxylin and eosin staining with or without immunohistochemistry for cytokeratin in accordance with previously published recommendations [16]. ALNs were considered positive if metastatic foci were $>0.2 \mathrm{~mm}$ and/or $>200$ tumor cells ( $\geq$ ypN1mi) according to the AJCC Cancer Staging Manual, seventh edition [13]. Any nodes with isolated metastatic foci of $\leq 0.2 \mathrm{~mm}$ were considered negative [ypN0(i+)], but were not considered to have achieved a node pathologic complete response (pCR). Regarding primary tumor responses to $\mathrm{NCT}$, the absence of in situ or invasive carcinomas (ypT0) or residual in situ carcinoma alone without invasive disease (ypTis) constituted a tumor pCR.

\section{Statistical analysis}

The detection of charcoal-tattooed node was investigated and its association with the detection of SLNs was analyzed. Detection failure of SLNs was defined as an inability to iden- 
Table 1. Clinicipathological characteristics of the enrolled patients

Parameter

Age (yr)

$$
\leq 50
$$

$>50$

BMI $\left(\mathrm{kg} / \mathrm{m}^{2}\right)$

$$
\leq 25
$$

$>25$

Laterality

Left

Right

$7(35.0)$

Location of the main lesion

Upper outer quadrant

Upper inner quadrant

Upper central area

Mediocentral area

Lower outer quadrant

Lower inner quadrant

Lower central area

Subareolar area

Histologic type

IDC-NOS

Mucinous carcinoma

$1(5.0)$

Clinical tumor stage at diagnosis

$\begin{array}{lr}\text { cT1 } & 6(30.0) \\ \text { cT2 } & 11(55.0) \\ \text { cT3 } & 3(15.0)\end{array}$

\section{Clinical node stage at diagnosis}

$\begin{array}{rr}\mathrm{cN} 1 & 12(60.0) \\ \mathrm{cN} 2 & 5(25.0) \\ \mathrm{cN} 3 & 3(15.0)\end{array}$

Regimens of NCT

$\mathrm{AC}-\mathrm{wP} \pm \mathrm{H}$

$9(45.0)$

$\mathrm{AC}-\mathrm{T} \pm \mathrm{H}$

$11(55.0)$

Tumor response to NCT

Response 14 (70.0)

Non-response

$6(30.0)$

Node response to NCT

Response

$11(55.0)$

Non-response

$9(45.0)$

Pathologic tumor stage

ypT0-is $6(30.0)$

ypT1-2 $14(70.0)$

Pathologic node stage

ypN0 $10(50.0)$

ypN1-2

$10(50.0)$

Pathologic TNM stage

Stage $0 \quad 6(30.0)$

Stage $1 \quad 4(20.0)$

Stage $2 \quad 7(35.0)$

Stage 3 3(15.0)

(Continued)
Table 1. Continued

Parameter

No. of patients (\%)

Histologic grade

I

II

$3(15.0)$

$13(65.0)$

III

$4(20.0)$

Lymphovascular invasion

Absent $15(75.0)$

Present 5 (25.0)

Perinodal extension

$\begin{array}{lr}\text { Absent } & 15(75.0) \\ \text { Present } & 5(25.0)\end{array}$

Present

$5(25.0)$

Estrogen receptor

Negative

$6(30.0)$

Positive

$14(70.0)$

Progesterone receptor

Negative $8(40.0)$

Positive $12(60.0)$

HER2

Negative $\quad 15(75.0)$

Positive 5 (25.0)

Molecular phenotype

Luminal A 10 (50.0)

Luminal B 4 (20.0)

HER2-enriched 2 (10.0)

TNBC $4(20.0)$

Type of breast surgery

Breast-conserving surgery $\quad 11(55.0)$

Total mastectomy $9(45.0)$

Type of axillary surgery

SLNB alone $2(10.0)$

SLNB+axillary sampling $\quad 6(30.0)$

SLNB+ALND $12(60.0)$

BMI, body mass index; IDC-NOS, invasive ductal carcinoma-not otherwise specified; NCT, neoadjuvant chemotherapy; AC, anthracycline followed by cyclophosphamide; wP, weekly paclitaxel; $\mathrm{H}$, trastuzumab; $\mathrm{T}$, docetaxel; TNM, tumor node metastasis; HER2, human epidermal growth factor receptor 2; TNBC, triple-negative breast cancer; SLNB, sentinel lymph node biopsy; ALND, axillary lymph node dissection.

tify hot and/ or blue nodes by lymphoscintigraphy or gamma probe with visual inspection. Sensitivity was calculated by dividing true positive (TP) findings by TP plus false-negative (FN) findings. FNR was defined as the proportion of patients with negative SLNs who subsequently had metastatic ALNs among patients with $\geq 1$ positive lymph node. NPV was calculated by dividing true negative (TN) findings by TN plus 
Table 2. Results of SLNB and axillary surgery

\begin{tabular}{|c|c|c|c|c|}
\hline \multirow{3}{*}{ Parameter } & \multicolumn{3}{|c|}{ Final ALN status after NCT } & \multirow{3}{*}{ Total } \\
\hline & \multicolumn{2}{|c|}{ Positive (n=10) } & \multirow{2}{*}{ Negative $(n=10)$} & \\
\hline & Non-sentinel ALN (+) & Non-sentinel ALN (-) & & \\
\hline \multicolumn{5}{|l|}{ SLNB } \\
\hline Positive & 3 & 5 & - & 8 \\
\hline Negative & 2 & - & 10 & 12 \\
\hline \multicolumn{5}{|c|}{ Modified SLNB ${ }^{\text {a) }}$} \\
\hline Positive & 4 & 6 & - & 10 \\
\hline Negative & 0 & - & 10 & 10 \\
\hline
\end{tabular}

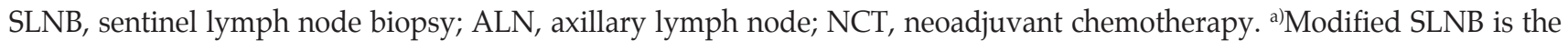
final results of hot or blue-colored sentinel lymph nodes and initial cytology-proven, charcoal-tattooed lymph node excised during axillary sampling procedure.

FN findings. Accuracy was defined as the proportion of patients with TP or TN among patients with successful SLNB. The significances of differences in SLNB performance of subgroups and in clinicopathological parameters between groups were evaluated using the chi-square test or Fisher exact test. The Mann-Whitney U test was used to compare the median values of continuous numerical data. All statistical tests were two-sided, and $\mathrm{p}<0.05$ was considered statistically significant. SPSS ver. 23.0 (IBM Corp., Armonk, NY) and SAS ver. 9.4 (SAS Inc., Cary, NC) were used for analysis.

\section{Ethical statement}

This study was approved by the Institutional Review Board (IRB) of Severance Hospital, Yonsei University Health System, Seoul, Korea (IRB No. 4-2015-0465) and registered to the Clinical Research Information Service (CRIS, http:// cris.nih.go.kr/cris / index.jsp; No. KCT0002370), Korea. Written informed consent was obtained from all patients prior to study entry.

\section{Results}

\section{Patient characteristics and ultrasound-guided charcoal tattooing procedure}

The mean age of 20 patients was $52.3 \pm 10.9$ years (range, 34 to 70 years) at diagnosis. The mean size of the main primary tumor at presentation was $3.1 \pm 1.8 \mathrm{~cm}$ (range, 1.2 to $8.4 \mathrm{~cm}$ ). Table 1 shows the clinicopathological characteristics and treatment patterns of the enrolled patients. No significant side effects or complications occurred during charcoal tattooing of metastatic ALN. After finishing the tattooing procedure, 16 patients $(80 \%)$ underwent NCT on the very day that charcoal tattooing was performed and the time interval between charcoal tattooing and NCT ranged from 0 to 5 days. The median time interval between charcoal tattooing and surgery was 181 days (range, 165 to 197 days).

Final permanent pathology of breast and ALNs is presented in Table 1. Preoperative imaging studies of three patients with initially suspicious non-axillary regional lymph nodes showed no radiological evidence of non-axillary regional metastasis after completion of NCT. Therefore, when pathologic results of the breast and axilla were considered, both tumor and nodal pCR were determined in six patients $(30.0 \%)$. There was no statistical difference in the nodal pCR rate according to the type of axillary surgery (ALND 41.7\% vs. axillary sampling 62.5\%, p=0.650) (Table 1).

\section{Axillary surgery and diagnostic performance of SLNB}

One or more hot and/or blue SLNs were detected in all patients (detection rate, 100\%). The median number of SLNs and total ALNs (SLNs+non-sentinel ALNs) retrieved was 3.0 (range, 1 to 12 ) and 10.0 (range, 4 to 18), respectively. The median number of non-sentinel ALNs excised was 4.5 (range, 0 to 15). The number of pathologically retrieved nodes in two patients who underwent SLNB alone was four and 10, respectively. During axillary surgery, charcoal-tattooed ALN and track were easily identified by visual inspection and excised in all patients (detection rate of tattooed node, 100\%). According to the type of axillary surgery, the median number of SLNs was not different $(n=3$ in each group, $p=0.624)$, but the median number of non-sentinel 
Table 3. Diagnostic performance of SLNB in all and subgroups of patients

\begin{tabular}{|c|c|c|c|c|}
\hline Parameter & Sensitivity $(\%)$ & FNR (\%) & NPV (\%) & Accuracy (\%) \\
\hline Overall $(n=20)$ & 80.0 & 20.0 & 83.3 & 90.0 \\
\hline $95 \% \mathrm{CI}$ & $55.2-100.0$ & $0.0-44.8$ & $62.2-100.0$ & $76.9-100.0$ \\
\hline \multicolumn{5}{|l|}{ No. of retrieved SLNs } \\
\hline$\leq 2(\mathrm{n}=2)$ & 0.0 & 100.0 & 50.0 & 50.0 \\
\hline $3(n=11)$ & 75.0 & 25.0 & 87.5 & 90.9 \\
\hline$\geq 4(\mathrm{n}=7)$ & 100.0 & 0.0 & 100.0 & 100.0 \\
\hline $\mathrm{p}$-value & 0.089 & 0.089 & 0.576 & 0.305 \\
\hline \multicolumn{5}{|l|}{ Type of axillary surgery } \\
\hline Axillary sampling $(\mathrm{n}=8)$ & 66.7 & 33.4 & 83.3 & 87.5 \\
\hline $\operatorname{ALND}(\mathrm{n}=12)$ & 85.7 & 14.3 & 83.3 & 91.7 \\
\hline $\mathrm{p}$-value & $>0.999$ & $>0.999$ & $>0.999$ & $>0.999$ \\
\hline \multicolumn{5}{|l|}{ Node response to NCT } \\
\hline Response ( $\mathrm{n}=11)$ & 100.0 & 0.0 & 100.0 & 100.0 \\
\hline Non-response $(\mathrm{n}=9)$ & 71.4 & 28.6 & 50.0 & 77.8 \\
\hline $\mathrm{p}$-value & $>0.999$ & $>0.999$ & 0.091 & 0.190 \\
\hline \multicolumn{5}{|l|}{ Molecular phenotype } \\
\hline Luminal $(\mathrm{n}=14)$ & 100.0 & 0.0 & 100.0 & 100.0 \\
\hline Non-luminal $(\mathrm{n}=6)$ & 33.3 & 66.7 & 60.0 & 66.7 \\
\hline $\mathrm{p}$-value & 0.067 & 0.067 & 0.152 & 0.079 \\
\hline
\end{tabular}

SLNB, sentinel lymph node biopsy; FNR, false-negative rate; NPV, negative predictive value; CI, confidence interval; SLNs, sentinel lymph nodes; ALND, axillary lymph node dissection; NCT, neoadjuvant chemotherapy.
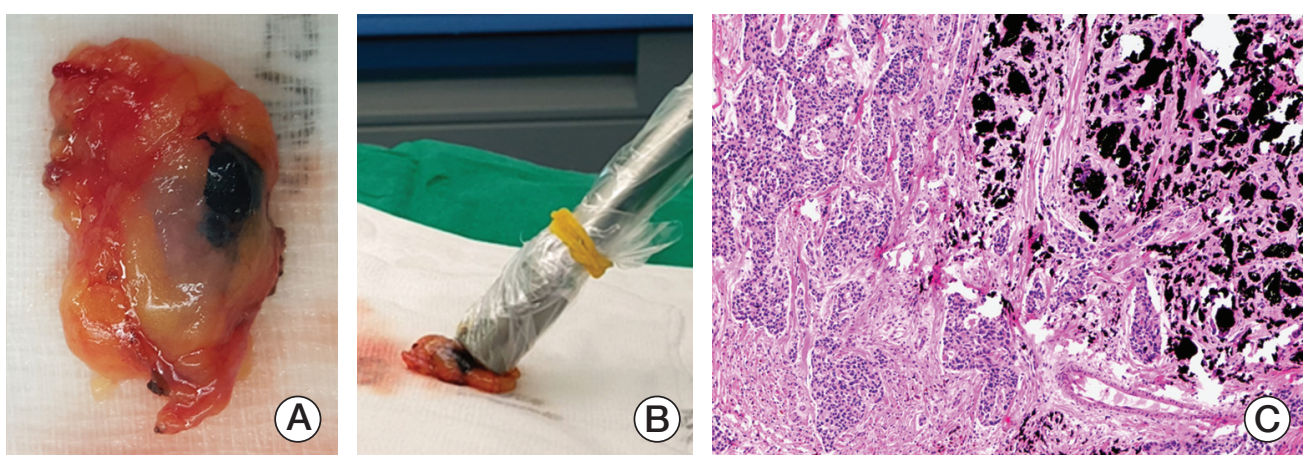

Fig. 1. Gross and microscopic findings of a charcoal-tattooed lymph node in a patient presenting with a false-negative sentinel lymph node biopsy using dual tracers. (A) Gross picture of a retrieved charcoal-tattooed axillary lymph node. (B) Ex vivo radioisotope counts of the tattooed node showing minimal uptake (value, 35). (C) Microscopic photograph of residual metastatic carcinomas and charcoal pigments in the tattooed axillary node (H\&E staining, $\times 100)$.

ALNs was significantly larger in the ALND group; therefore, the median number of total ALNs removed was significantly higher in the ALND group (ALND, 13 [range, 7 to 18] vs. axillary sampling, 4.5 [range, 4 to 10]; $\mathrm{p}<0.001$ ).

Table 2 compares SLNB results of removing hot and / or blue SLNs with the final ALN status. In addition to hot and/or blue SLNs, charcoal-tattooed node was calculated together as the modified SLNB. The diagnostic performance of the modified SLNB showed the highest values as follows: sensitivity, 100\%; FNR, 0.0\%; NPV, 100\%; and accuracy, $100 \%$.

Overall diagnostic performance of SLNB is presented in 
Table 4. Clinicopathological characteristics between patients whose SLNs concordantly included a charcoal-tattooed lymph node and those not

\begin{tabular}{|c|c|c|c|}
\hline Parameter & Concordant ( $n=15)$ & Discordant $(n=5)$ & p-value ${ }^{a)}$ \\
\hline \multicolumn{4}{|l|}{ Age (yr) } \\
\hline$\leq 50$ & $10(66.7)$ & $1(20.0)$ & 0.127 \\
\hline$>50$ & $5(33.3)$ & $4(80.0)$ & \\
\hline \multicolumn{4}{|l|}{ BMI $\left(\mathrm{kg} / \mathrm{m}^{2}\right)$} \\
\hline$\leq 25$ & $12(80.0)$ & $3(60.0)$ & 0.560 \\
\hline$>25$ & $3(20.0)$ & $2(40.0)$ & \\
\hline \multicolumn{4}{|l|}{ Laterality } \\
\hline Left & $10(66.7)$ & $3(60.0)$ & $>0.999$ \\
\hline Right & $5(33.3)$ & $2(40.0)$ & \\
\hline \multicolumn{4}{|c|}{ Clinical tumor stage at diagnosis } \\
\hline cT1 & $3(20.0)$ & $3(60.0)$ & 0.131 \\
\hline cT2-3 & $12(80.0)$ & $2(40.0)$ & \\
\hline \multicolumn{4}{|c|}{ Clinical node stage at diagnosis } \\
\hline cN1 & $10(66.7)$ & $2(40.0)$ & 0.347 \\
\hline $\mathrm{cN} 2-3$ & $5(33.3)$ & $3(60.0)$ & \\
\hline \multicolumn{4}{|l|}{ Regimens of NCT } \\
\hline $\mathrm{AC}-\mathrm{wP} \pm \mathrm{H}$ & $7(46.7)$ & $2(40.0)$ & $>0.999$ \\
\hline $\mathrm{AC}-\mathrm{T} \pm \mathrm{H}$ & $8(53.3)$ & $3(60.0)$ & \\
\hline \multicolumn{4}{|c|}{ Tumor response to NCT } \\
\hline Response & $10(66.7)$ & $4(80.0)$ & $>0.999$ \\
\hline Non-response & $5(33.3)$ & $1(20.0)$ & \\
\hline \multicolumn{4}{|c|}{ Node response to NCT } \\
\hline Response & $9(60.0)$ & $2(40.0)$ & 0.617 \\
\hline Non-response & $6(40.0)$ & $3(60.0)$ & \\
\hline \multicolumn{4}{|c|}{ Pathologic tumor stage } \\
\hline ypT0-is & $4(26.7)$ & $2(40.0)$ & 0.613 \\
\hline урT1-2 & $11(73.3)$ & $3(60.0)$ & \\
\hline \multicolumn{4}{|c|}{ Pathologic node stage } \\
\hline ypN0 & $7(46.7)$ & $3(60.0)$ & $>0.999$ \\
\hline ypN1-2 & $8(53.3)$ & $2(40.0)$ & \\
\hline \multicolumn{4}{|c|}{ Pathologic TNM stage } \\
\hline Stage 0 & $4(26.7)$ & $2(40.0)$ & 0.613 \\
\hline Stage 1-3 & $11(73.3)$ & $3(60.0)$ & \\
\hline \multicolumn{4}{|l|}{ Histologic grade } \\
\hline $\mathrm{I} / \mathrm{II}$ & $12(80.0)$ & $4(80.0)$ & $>0.999$ \\
\hline III & $3(20.0)$ & $1(20.0)$ & \\
\hline \multicolumn{4}{|c|}{ Lymphovascular invasion } \\
\hline Absent & $11(73.3)$ & $4(80.0)$ & $>0.999$ \\
\hline Present & $4(26.7)$ & $1(20.0)$ & \\
\hline \multicolumn{4}{|c|}{ Perinodal extension } \\
\hline Absent & $11(73.3)$ & $4(80.0)$ & $>0.999$ \\
\hline Present & $4(26.7)$ & $1(20.0)$ & \\
\hline \multicolumn{4}{|l|}{ Estrogen receptor } \\
\hline Negative & $3(20.0)$ & $3(60.0)$ & 0.131 \\
\hline Positive & $12(80.0)$ & $2(40.0)$ & \\
\hline \multicolumn{4}{|c|}{ Progesterone receptor } \\
\hline Negative & $5(33.3)$ & $3(60.0)$ & 0.347 \\
\hline Positive & $10(66.7)$ & $2(40.0)$ & \\
\hline
\end{tabular}

(Continued to the next page) 
Table 4. Continued

\begin{tabular}{|c|c|c|c|}
\hline Parameter & Concordant $(n=15)$ & Discordant $(n=5)$ & p-value ${ }^{a)}$ \\
\hline \multicolumn{4}{|l|}{ HER2 } \\
\hline Negative & $11(73.3)$ & $4(80.0)$ & $>0.999$ \\
\hline Positive & $4(26.7)$ & $1(20.0)$ & \\
\hline \multicolumn{4}{|l|}{ Molecular phenotype } \\
\hline Luminal A & $8(53.3)$ & $2(40.0)$ & 0.319 \\
\hline Luminal B & $4(26.7)$ & 0 & \\
\hline HER2-enriched & $1(6.7)$ & $1(20.0)$ & \\
\hline TNBC & $2(13.3)$ & $2(40.0)$ & \\
\hline \multicolumn{4}{|l|}{ Type of breast surgery } \\
\hline Breast-conserving surgery & $9(60.0)$ & $2(40.0)$ & 0.617 \\
\hline Total mastectomy & $6(40.0)$ & $3(60.0)$ & \\
\hline \multicolumn{4}{|l|}{ Type of axillary surgery } \\
\hline SLNB \pm axillary sampling & $6(40.0)$ & $2(40.0)$ & $>0.999$ \\
\hline SLNB+ALND & $9(60.0)$ & $3(60.0)$ & \\
\hline
\end{tabular}

SLN, sentinel lymph node; BMI, body mass index; NCT, neoadjuvant chemotherapy; AC, anthracycline followed by cyclophosphamide; wP, weekly paclitaxel; H, trastuzumab; T, docetaxel; HER2, human epidermal growth factor receptor 2; TNBC, triple-negative breast cancer; SLNB, sentinel lymph node biopsy; ALND, axillary lymph node dissection. ${ }^{a} \mathrm{p}$-value was calculated by the Fisher exact test.

Table 3 including subgroups. When more SLNs were excised, the sensitivity, NPV, and accuracy improved and FNR decreased, although statistical significance was not demonstrated. Similarly, wider axillary surgery or favorable nodal response was associated with improved diagnostic performance of SLNB. Better performance was exhibited in patients with luminal A or B subtypes than in those with non-luminal (HER2-enriched or TNBC) subtypes with borderline significance.

\section{Association between sentinel and tattooed lymph nodes}

Charcoal-tattooed ALN was among the hot and/or blue SLNs in 15 patients (75.0\%) but removed during cold and non-blue axillary nodes sampling in five cases (25.0\%). Among 15 patients with concordance between the tattooed node and SLNs, eight (53.3\%) showed residual metastatic disease in the charcoal-tattooed ALN. The other seven patients $(46.7 \%)$ were determined to have negative conversion of the tattooed node and finally achieved nodal PCR. Among five patients with discordance between the tattooed ALN and SLNs, three (60.0\%) showed negative sentinel and tattooed nodes and finally achieved nodal pCR. Of the remaining two patients, one 61-year-old patient showed that three SLNs detected were negative, but the charcoal-tattooed non-sentinel ALN showed residual metastasis (Fig. 1). In the other 59-year-old patient, only one SLN was retrieved. Frozen section and permanent pathology of the SLN con- firmed the presence of a few atypical cells and isolated tumor cells with a maximal diameter of $100 \mu \mathrm{m}$ [ypNO(i+)], respectively. Subsequently, ALND was performed and metastatic carcinomas in four out of 12 ALNs were found including micrometastasis $(450 \mu \mathrm{m})$ in the tattooed ALN.

Regarding the intensity order of the uptaken radioisotope, the tattooed node was the hottest in eight patients, the second hottest in three, and the third hottest in four. When clinicopathological characteristics of patients with concordant SLNs and tattooed node were compared to those of patients with discordant findings, no statistically significant parameters were identified (Table 4).

\section{Discussion}

In patients with clinically or histopathologically node-positive disease at presentation, SLNB can decrease morbidity while increasing quality of life by allowing ALND to be omitted when the axilla is converted to clinically node-negative status after $\mathrm{NCT}$, similar benefits to those proven in initially node-negative patients $[17,18]$. However, the diagnostic performance and oncological safety of SLNB has been evolving and long-term follow-up results are required to clearly define the role of SLNB in these patients. Recently, the SENTINA, ACOSOG Z1071, and SN FNAC trials showed SLNB being 

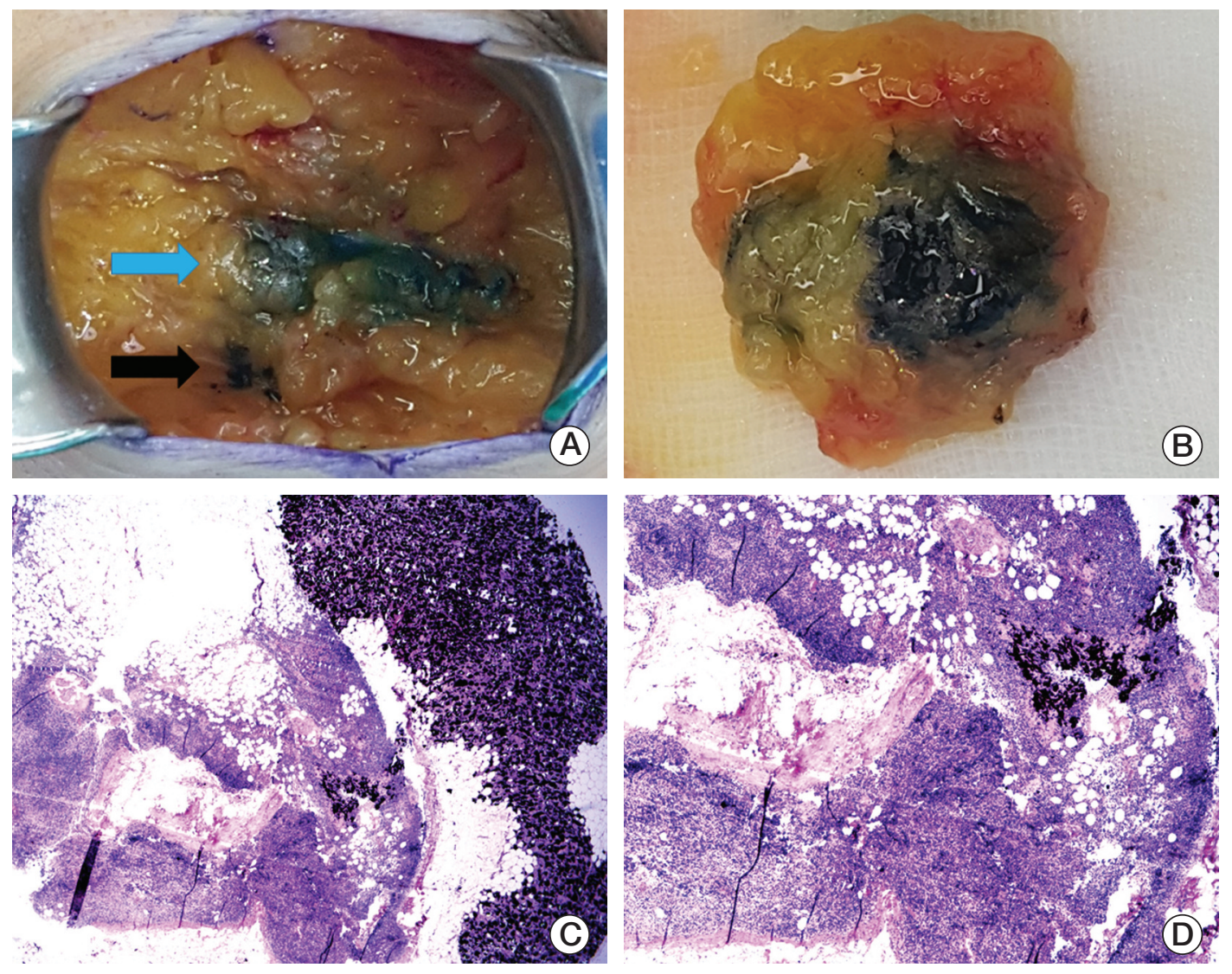

Fig. 2. Intraoperative photographs and pathologic slides of a sentinel lymph node. (A) Charcoal tattoo (black arrow) and blue dye (blue arrow) tracks during axillary surgery. (B) Excised sentinel node marked with the tattoo and blue dye. Lowpower field $(H \& E$ staining, $\times 20)(C)$ and high-power field $(H \& E$ staining, $\times 100)(D)$ microscopic views show tattoo pigments with no residual metastatic carcinoma in the sentinel node.

performed in this setting under somewhat different protocols and the results of these studies found the following technical issues to be conclusively associated with SLNB accuracy: marking of biopsied ALNs, use of dual tracers, numbers of SLNs retrieved, and immunohistochemical evaluation [4,19]. The National Comprehensive Cancer Network guidelines recommend either type of axillary surgery as a category $2 \mathrm{~B}$ and also state the technical considerations [9].

In the present study, hot and/or blue SLNs as well as black-tattooed ALN and track could be detected in all patients after NCT within a median duration of 6 months, fulfilling the technical feasibility of node tattooing without significant morbidity. Similarly, black ink could be identified intraoperatively up to an average 130 days after tattooing in a report of Choy et al. [11]. Three or more SLNs were removed in 18 patients $(90.0 \%)$ and the tattooed ALN was included in the SLNs of 15 patients (75.0\%). On gross inspection, the blue dye of the black tattooed nodes was faint in some cases [11], but subtle different coloring or the direction of the running tracks guided discrimination between sentinel and non-sentinel nodes with the assistance of radioisotopes (Fig. 2). Pathologic examination was not encumbered by the black pigments on the slides.

More importantly, improved diagnostic performance of SLNB was again demonstrated by marking of metastatic node with charcoal tattoo even small size of this study and inability to calculate the number of $\mathrm{TN}$ due to $40 \%$ of our cases without ALND. In addition, invasive localization procedure is not required preoperatively and it is not necessary to verify removal of a clip or radioactive seed in surgical specimen during surgery using specimen mammography. In patients with much favorable nodal response to NCT who are a potential candidate of SLNB, even specialized radiologists may have difficulty to detect a clip in an ALN by ultrasound and mammographic detection or targeting might be performed before surgery. However, black-color charcoal 

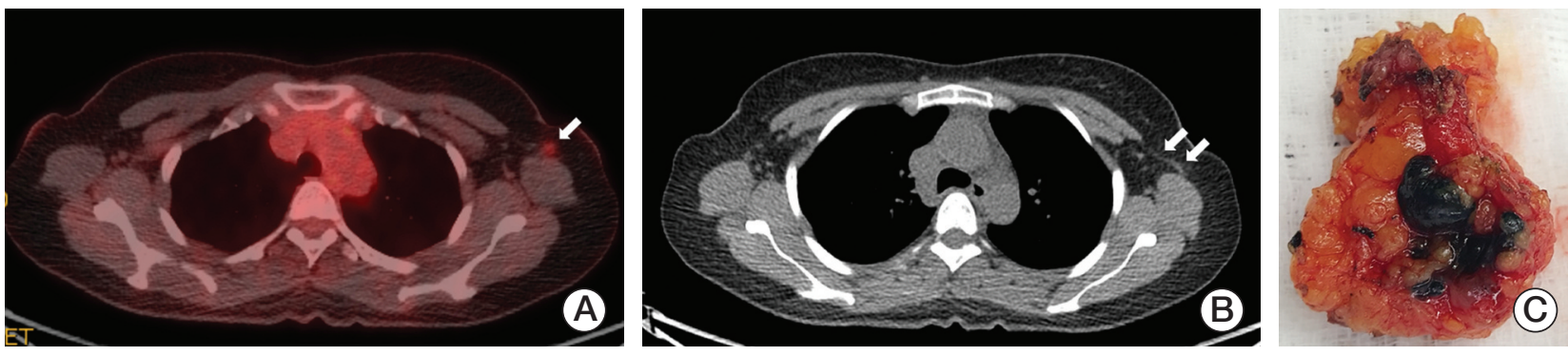

Fig. 3. Images and tattooed sentinel node of a patient with an axillary arch. (A) The ${ }^{18} \mathrm{~F}$-fluorodeoxyglucose (FDG) positron emission tomography-computed tomography (PET-CT) scan shows increased FDG uptake by the metastatic axillary lymph node in the level I left axilla (arrow). (B) The PET-CT scan demonstrates the left axillary arch that is also known as the axillopectoral muscle (double arrow). (C) The hot and tattooed sentinel lymph node is retrieved.

was easily detected during surgery without any additional mechanical device or equipment. Clinically negligible risk of charcoal migration or absorption was presented in this study and additional radiation hazard did not exist. However, long-term benefits and complications of charcoal tattooing should be further validated using large cohort studies.

According to predefined SLNB and pathological node response, the FNR of this study with a small cohort was worse than that of a prior meta-analyses [20,21]. However, when the protocols of the SN FNAC study were followed, the performance of SLNB was comparable when ypNO(i+) was considered node-positive [8]. Although the clinical relevance of very small residual nodal disease after NCT remains unclear [22], the more number of SLNs retrieved or the wider type of axillary surgery increased the accuracy of SLNB. Similar to this study, Mamtani et al. [23] reported that at least three SLNs including abnormally palpable nodes during surgery could be removed in $86 \%$ of patients, but the SENTINA and ACOSOG Z1071 studies reported that approximately one-third to one-half of patients could have $\geq 3$ SLNs retrieved, respectively [6,7]. It is challenging to determine whether $\geq 3$ SLNs can be retrieved in the majority of patients after NCT.

Persistently suspicious ALN metastasis after completion of NCT is not an indication for SLNB in initially node-positive patients $[9,18]$. In the ACOSOG Z1071 trial, approximately $70 \%$ of patients presented normalized nodal features on axillary ultrasound after NCT and these patients showed acceptable SLNB performance with a FNR of 9.8\% [24]. Current cohorts with favorable nodal response to NCT seen on preoperative work-ups also demonstrated better performance of SLNB with tattooing. Therefore, axillary restaging by image modalities after NCT could inform patient selection and further improvements of optimal techniques should be investigated in this setting [19]. In addition, molecular phe- notypes are well-known predictive factors of NCT response. Although the small sample size in this study was not conclusive, luminal subtypes showed better performance than nonluminal subtypes in contrast to findings from prior studies $[3,25]$. More research is needed to answer the remaining questions.

Basically, SLNB should be used to excise hot and/or blue nodes detected by dual tracers as well as suspicious enlarged cold and non-blue ALNs intraoperatively in both adjuvant and neoadjuvant settings [26,27]. In the present study, 11 patients underwent suspicious ALN sampling including five tattooed node excisions and of them, three (27.3\%) showed additional two residual macronodal and one residual micronodal metastasis from the sampled ALNs. According to the practically recommended SLNB technique, the performance of the modified SLNB in this study was the best. Our results again emphasize the removal of suspiciously cold or non-blue ALNs during SLNB. However, considering the chance of non-palpable partial nodal response to NCT and the need for balance between surgical morbidity and diagnostic accuracy, charcoal marking at diagnosis followed by excision of the biopsy-proven ALN after NCT, so called targeted axillary dissection, can be one of the best ways to improve the performance in concordance with suggestions from the ACOSOG Z1071 study [10,28]. Furthermore, there was no significant difference in clinicopathological parameters between patients with tattooed ALN included in SLNB and axillary sampling, suggesting the importance of the marking technique itself to achieve successful targeted axillary dissection.

Patterns of lymphatic drainage to SLNs in breast cancer are known to be individual characteristic and are even detected in internal mammary chains by lymphoscintigraphy [29]. Langer's axillary arch, an anatomical variation, is found in $7 \%$ to $10 \%$ of cases during surgery or by preoperative diag- 
nosis and can affect SLNB [30]. A 47-year-old woman who underwent SLNB guided by only a radioisotope had a left axillary arch noted in her initial diagnostic work-up, but her tattooed node with the third intensity of modest uptake was unexpectedly located in the deep portion of the axilla during surgery, which might have been missed if done without caution (Fig. 3). Fortunately, guided by the tattoo track, the biopsy-proven tattooed SLN could be easily removed and technical mistakes were prevented. Although the clinical impact of the axillary arch on SLNB in this setting is currently uncertain, these rare circumstances can be happen to anyone and should be kept in mind.

In conclusion, our initial experience with charcoal tattooing of cytology-confirmed metastatic ALN at presentation showed technical feasibility without significant morbidity in breast cancer patients treated with NCT. SLNB using dual tracers after NCT is not limited by positive node tattooing and has been demonstrated to improve accuracy. Furthermore, invasive preoperative localization is not additionally required to detect the marked node. The tattooing procedure can prevent technical errors during SLNB. A multicenter study with a large study population is necessary to determine the clinical implications of the node-tattooing technique including long-term side effects and its oncological safety in this setting.

\section{Conflicts of Interest}

Conflict of interest relevant to this article was not reported.

\section{Acknowledgments}

This study was supported by a faculty research grant of Yonsei University College of Medicine for 2015 (grant No. 6-2015-0161) and by the Ministry of Education of the Republic of Korea and the National Research Foundation of Korea (NRF-2015S1A5B8036349 and NRF-2017R1A2B4010407).

\section{References}

1. Lyman GH, Somerfield MR, Bosserman LD, Perkins CL, Weaver DL, Giuliano AE. Sentinel lymph node biopsy for patients with early-stage breast cancer: American Society of Clinical Oncology clinical practice guideline update. J Clin Oncol. 2017;35:561-4.

2. Bromham N, Schmidt-Hansen M, Astin M, Hasler E, Reed MW. Axillary treatment for operable primary breast cancer. Cochrane Database Syst Rev. 2017;1:CD004561.

3. Park S, Park JM, Cho JH, Park HS, Kim SI, Park BW. Sentinel lymph node biopsy after neoadjuvant chemotherapy in patients with cytologically proven node-positive breast cancer at diagnosis. Ann Surg Oncol. 2013;20:2858-65.

4. Pilewskie M, Morrow M. Axillary nodal management following neoadjuvant chemotherapy: a review. JAMA Oncol. 2017;3:549-55.

5. Jatoi I, Benson JR, Toi M. De-escalation of axillary surgery in early breast cancer. Lancet Oncol. 2016;17:e430-41.

6. Kuehn T, Bauerfeind I, Fehm T, Fleige B, Hausschild M, Helms $\mathrm{G}$, et al. Sentinel-lymph-node biopsy in patients with breast cancer before and after neoadjuvant chemotherapy (SENTINA): a prospective, multicentre cohort study. Lancet Oncol. 2013;14:609-18.

7. Boughey JC, Suman VJ, Mittendorf EA, Ahrendt GM, Wilke LG, Taback B, et al. Sentinel lymph node surgery after neoadjuvant chemotherapy in patients with node-positive breast cancer: the ACOSOG Z1071 (Alliance) clinical trial. JAMA. 2013;310:1455-61.

8. Boileau JF, Poirier B, Basik M, Holloway CM, Gaboury L, Sideris $\mathrm{L}$, et al. Sentinel node biopsy after neoadjuvant chemotherapy in biopsy-proven node-positive breast cancer: the SN FNAC study. J Clin Oncol. 2015;33:258-64.

9. Gradishar WJ, Anderson BO, Balassanian R, Blair SL, Burstein HJ, Cyr A, et al. Invasive breast cancer version 1.2016, NCCN clinical practice guidelines in oncology. J Natl Compr Canc Netw. 2016;14:324-54.

10. Caudle AS, Yang WT, Krishnamurthy S, Mittendorf EA, Black DM, Gilcrease MZ, et al. Improved axillary evaluation following neoadjuvant therapy for patients with node-positive breast cancer using selective evaluation of clipped nodes: implementation of targeted axillary dissection. J Clin Oncol. 2016;34: 1072-8.

11. Choy N, Lipson J, Porter C, Ozawa M, Kieryn A, Pal S, et al. Initial results with preoperative tattooing of biopsied axillary lymph nodes and correlation to sentinel lymph nodes in breast cancer patients. Ann Surg Oncol. 2015;22:377-82.

12. Shin K, Caudle AS, Kuerer HM, Santiago L, Candelaria RP, Dogan B, et al. Radiologic mapping for targeted axillary dissection: needle biopsy to excision. AJR Am J Roentgenol. 2016;207:1372-9.

13. Edge SB, Byrd DR, Compton CC, Fritz AG, Greene FL, Trotti A. AJCC cancer staging manual. 7th ed. New York: Springer; 2010.

14. Hammond ME, Hayes DF, Dowsett M, Allred DC, Hagerty KL, Badve S, et al. American Society of Clinical Oncology/College Of American Pathologists guideline recommendations for immunohistochemical testing of estrogen and progesterone receptors in breast cancer. J Clin Oncol. 2010;28:2784-95.

15. Wolff AC, Hammond ME, Hicks DG, Dowsett M, McShane 
LM, Allison $\mathrm{KH}$, et al. Recommendations for human epidermal growth factor receptor 2 testing in breast cancer: American Society of Clinical Oncology/College of American Pathologists clinical practice guideline update. J Clin Oncol. 2013;31: 3997-4013.

16. Weaver DL. Pathology evaluation of sentinel lymph nodes in breast cancer: protocol recommendations and rationale. Mod Pathol. 2010;23 Suppl 2:S26-32.

17. Glechner A, Wockel A, Gartlehner G, Thaler K, Strobelberger M, Griebler U, et al. Sentinel lymph node dissection only versus complete axillary lymph node dissection in early invasive breast cancer: a systematic review and meta-analysis. Eur J Cancer. 2013;49:812-25.

18. Mastrangelo S, McMasters K, Ajkay N. Surgical management of the axilla in breast cancer. Am Surg. 2016;82:475-86.

19. Rubio IT. Sentinel lymph node biopsy after neoadjuvant treatment in breast cancer: work in progress. Eur J Surg Oncol. 2016;42:326-32.

20. van Nijnatten TJ, Schipper RJ, Lobbes MB, Nelemans PJ, BeetsTan RG, Smidt ML. The diagnostic performance of sentinel lymph node biopsy in pathologically confirmed node positive breast cancer patients after neoadjuvant systemic therapy: a systematic review and meta-analysis. Eur J Surg Oncol. 2015;41:1278-87.

21. El Hage Chehade H, Headon H, El Tokhy O, Heeney J, Kasem A, Mokbel K. Is sentinel lymph node biopsy a viable alternative to complete axillary dissection following neoadjuvant chemotherapy in women with node-positive breast cancer at diagnosis? An updated meta-analysis involving 3,398 patients. Am J Surg. 2016;212:969-81.

22. King TA, Morrow M. Surgical issues in patients with breast cancer receiving neoadjuvant chemotherapy. Nat Rev Clin Oncol. 2015;12:335-43.

23. Mamtani A, Barrio AV, King TA, Van Zee KJ, Plitas G, Pilewskie $\mathrm{M}$, et al. How often does neoadjuvant chemotherapy avoid axillary dissection in patients with histologically con- firmed nodal metastases? Results of a prospective study. Ann Surg Oncol. 2016;23:3467-74.

24. Boughey JC, Ballman KV, Hunt KK, McCall LM, Mittendorf EA, Ahrendt GM, et al. Axillary ultrasound after neoadjuvant chemotherapy and its impact on sentinel lymph node surgery: results from the American College of Surgeons Oncology Group Z1071 Trial (Alliance). J Clin Oncol. 2015;33:3386-93.

25. Enokido K, Watanabe C, Nakamura S, Ogiya A, Osako T, Akiyama F, et al. Sentinel lymph node biopsy after neoadjuvant chemotherapy in patients with an initial diagnosis of cytology-proven lymph node-positive breast cancer. Clin Breast Cancer. 2016;16:299-304.

26. Lyman GH, Giuliano AE, Somerfield MR, Benson AB 3rd, Bodurka DC, Burstein HJ, et al. American Society of Clinical Oncology guideline recommendations for sentinel lymph node biopsy in early-stage breast cancer. J Clin Oncol. 2005;23: 7703-20.

27. Amersi F, Giuliano AE. Management of the axilla. Hematol Oncol Clin North Am. 2013;27:687-702.

28. Boughey JC, Ballman KV, Le-Petross HT, McCall LM, Mittendorf EA, Ahrendt GM, et al. Identification and resection of clipped node decreases the false-negative rate of sentinel lymph node surgery in patients presenting with node-positive breast cancer (T0-T4, N1-N2) who receive neoadjuvant chemotherapy: results From ACOSOG Z1071 (Alliance). Ann Surg. 2016;263:802-7.

29. Kawase K, Gayed IW, Hunt KK, Kuerer HM, Akins J, Yi M, et al. Use of lymphoscintigraphy defines lymphatic drainage patterns before sentinel lymph node biopsy for breast cancer. J Am Coll Surg. 2006;203:64-72.

30. Ando J, Kitamura T, Kuroki Y, Igarashi S. Preoperative diagnosis of the axillary arch with multidetector row computed tomography and the axillary arch in association with anatomical problems of sentinel lymph node biopsy. Breast Cancer. 2010;17:3-8. 